\title{
Room temperature excitation spectroscopy of single quantum dots
}

\author{
Christian Blum ${ }^{1, \S}$, Frank Schleifenbaum ${ }^{2, \S}$, Martijn Stopel ${ }^{1}$, \\ Sébastien Peter $^{2}$, Marcus Sackrow ${ }^{3,4}$, Vinod Subramaniam ${ }^{* 1}$ \\ and Alfred J. Meixner ${ }^{* 3}$
}

\section{Full Research Paper}

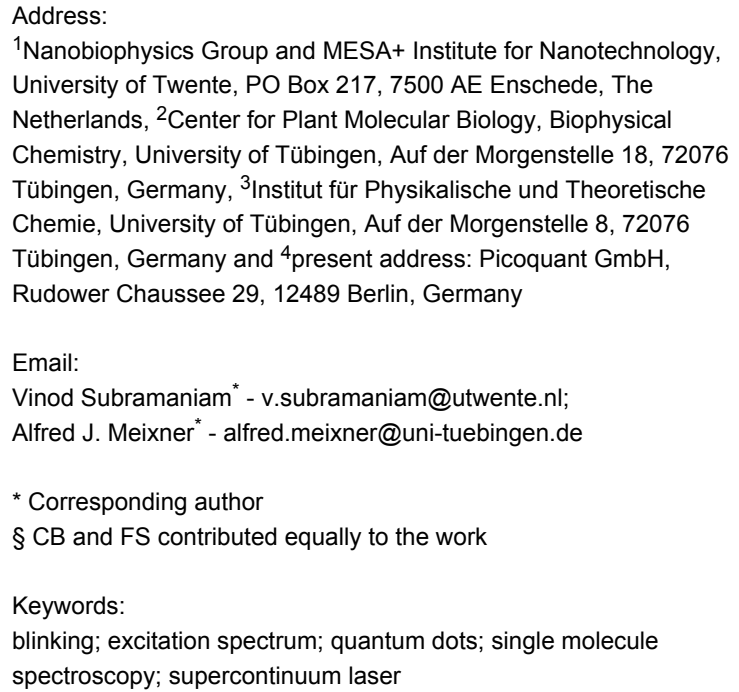

Beilstein J. Nanotechnol. 2011, 2, 516-524. doi:10.3762/bjnano.2.56

Received: 22 April 2011

Accepted: 09 June 2011

Published: 30 August 2011

This article is part of the Thematic Series "Nanophotonics, nano-optics and nanospectroscopy".

Guest Editor: A. J. Meixner

(C) 2011 Blum et al; licensee Beilstein-Institut. License and terms: see end of document.

\begin{abstract}
We report a single molecule detection scheme to investigate excitation spectra of single emitters at room temperature. We demonstrate the potential of single emitter photoluminescence excitation spectroscopy by recording excitation spectra of single CdSe nanocrystals over a wide spectral range of $100 \mathrm{~nm}$. The spectra exhibit emission intermittency, characteristic of single emitters. We observe large variations in the spectra close to the band edge, which represent the individual heterogeneity of the observed quantum dots. We also find specific excitation wavelengths for which the single quantum dots analyzed show an increased propensity for a transition to a long-lived dark state. We expect that the additional capability of recording excitation spectra at room temperature from single emitters will enable insights into the photophysics of emitters that so far have remained inaccessible.
\end{abstract}

\section{Introduction}

Since the first demonstration of single molecule fluorescence spectroscopy over two decades ago, techniques to detect and characterize the emission from single emitters have become increasingly sophisticated and versatile. These developments have made optical single molecule spectroscopy an indispensable tool to address complex problems in chemistry [1-3], in material sciences [4-6], and in life sciences [7-11]. 
A number of parameters that characterize single molecule emission are now routinely accessible at ambient temperatures, including emission intensity and polarization $[12,13]$, fluorescence lifetime [14-16], and the emission spectrum [17-19]. Access to these parameters yields unique insights into distinct properties of single molecules, and enables the determination of the distributions of the relevant experimental parameters, revealing, for example, distinct sub-states and energetic levels in a heterogeneous population [20,21]. Furthermore, external tailoring or directing of molecular emission has also been reported [22-24].

However, a detailed study of frequency resolved excitation dependent processes at the single molecule level, at room temperature, has not been experimentally achievable so far. Appreciation of these processes is fundamentally important for the understanding of the basic physics and for applications in next-generation photonic devices. The primary challenge has been the intrinsic difficulty in measuring the absorption of a single emitter at room temperature due to the extremely low signal to noise ratio. Although recent reports have demonstrated the detection of single molecule absorbance [25-27], a complete single molecule absorbance spectrum at room temperature has not yet been reported. A complementary approach to access the frequency dependent coupling of an emitter to an external electromagnetic field is based on photoluminescence excitation spectroscopy. Single emitter photoluminescence excitation microscopy has been already achieved in the early days of single molecule detection, but has been limited to experiments at cryogenic temperatures where the linewidths of individual emitters are not inhomogeneously broadened [28,29]. Hence, only a very limited excitation wavelength range was required to resolve individual absorbance properties of a single emitter at low temperatures.

In this paper, we describe the first successful acquisition of single emitter excitation spectra under ambient conditions over a wide spectral range. We combine a tunable white-light laser source with a confocal microscope with single molecule detection sensitivity to demonstrate excitation spectra of isolated semiconductor nanocrystals. These fluorophores, often referred to as quantum dots, have unique optical properties [30-35], including a narrow and tailored luminescence emission spectrum and significantly enhanced photostability compared to organic fluorophores. These properties make quantum dots promising nanomaterials in various fields of research, ranging from in vivo probes in the life-sciences [10,36,37] to single photon light sources in telecommunications [38] or quantum computing [30,35]. We demonstrate here how single emitter excitation spectroscopy provides a valuable addition to the range of single emitter spectroscopy techniques, yielding new insights into the complex photophysics of quantum dots.

The excitation spectrum, commonly used in ensemble fluorescence spectroscopy, depicts the evolution of the emission intensity recorded in a fixed spectral detection window upon scanning the excitation wavelength. Moreover, the excitation spectrum of an emitter coincides with its absorbance spectrum if the quantum efficiency is independent of excitation wavelength, which is generally assumed to be true for most emitters over large wavelength ranges. Hence, measurement of the excitation spectra of individual quantum dots permits access to the individual absorbance properties that are not accessible by common single molecule techniques or not at all by ensemble approaches.

\section{Results and Discussion}

In this study, we recorded excitation spectra of 48 individual $\mathrm{CdSe} / \mathrm{ZnS}$ core-shell quantum dots at room temperature. Since the occurrence of emission intermittencies (blinking) is a clear indication for the observation of a single emitter, and because blinking of quantum dots is still not fully understood, we did not apply any measures to suppress or minimize blinking. The single quantum dot excitation spectra recorded exhibited the main characteristics of a declining slope from shorter to longer wavelengths, and a peak close to the band edge transition, which we identify as the $1 \mathrm{~S}(\mathrm{e})-2 \mathrm{~S}_{3 / 2}(\mathrm{~h})$ transition [39]. However, we find distinct differences in the individual spectra that can be attributed to individual photophysical properties of the analyzed single quantum dots as well as to the well-known transitions of single emitters to dark, non-emitting, states. A typical example of an excitation spectrum obtained from a single quantum dot is shown in Figure 1a. In contrast to the ensemble spectrum, we observed distinct dips and gaps in the single quantum dot excitation spectra, which in principle could either result from blinking or reflect the photophysical properties of the quantum dot.

Semiconductor quantum dots exhibit a discrete structure of quantized energy states. Hence, one would expect to observe discrete bands in both the excitation and absorbance spectra when the excitation wavelength is in resonance with a transition to such a discrete state. Low temperature experiments showed narrow emission lines [40], but also revealed that only a few sharp transitions in the direct vicinity of the band edge can be found, while at higher excitation energies the optical transitions merge into a dense quasicontinuum [41]. At room temperature, these sharp transitions experience inhomogeneous broadening effects, mainly due to lattice vibrations. It is therefore not surprising that no sharp transitions are resolved in the room temperature ensemble excitation spectra of quantum dots. 
a)

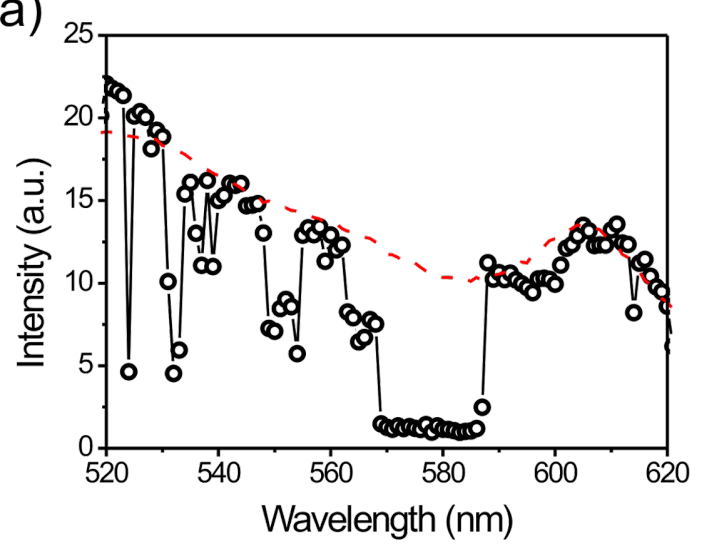

c)

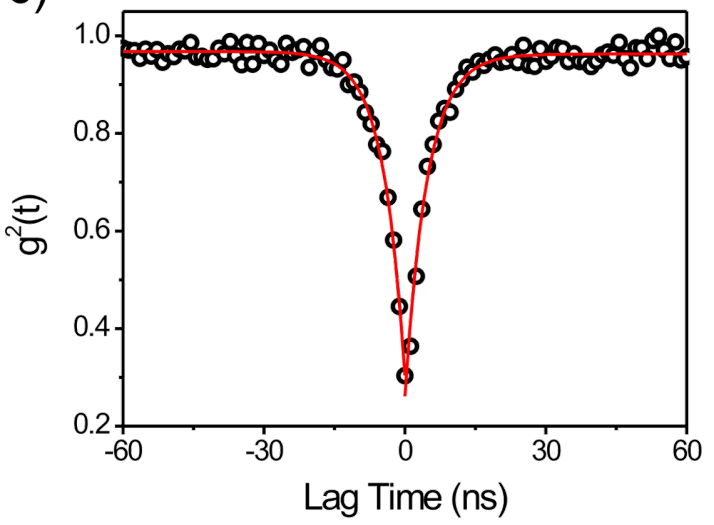

b)

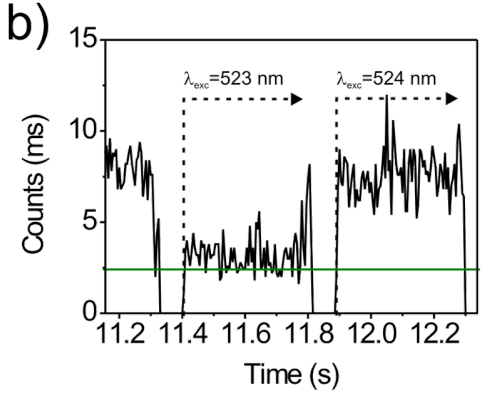

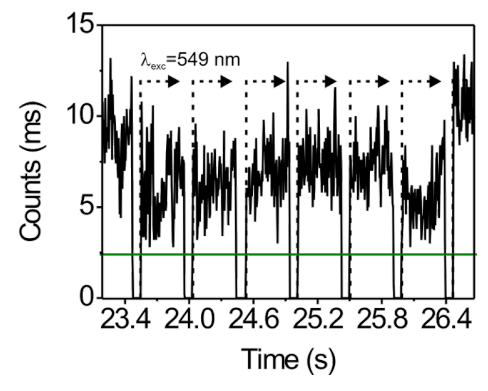

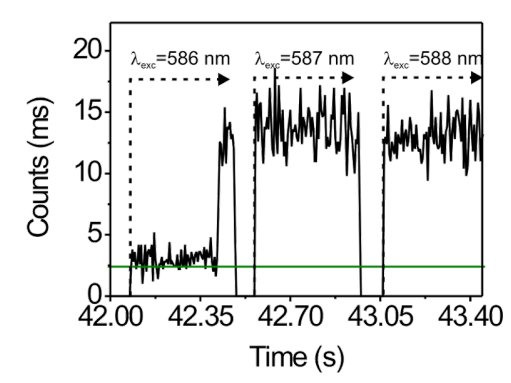

Figure 1: Single emitter characteristics observed by excitation spectroscopy of isolated quantum dots. a) Excitation spectrum of a single quantum dot (open circles) with typical intensity intermittencies that result in drops and gaps in the spectrum. The ensemble excitation spectrum is shown for comparison (red dashed line). b) Intensity trajectories of a single quantum dot for selected excitation wavelengths (green line marks the background level). c) Photon antibunching curve of a single quantum dot.

The spectra demonstrate obvious intensity fluctuations of different magnitudes within a single measurement interval, which are characteristic of the emission from a single emitter. These fluctuations have been reported for semiconductor quantum dots, and have only recently been circumvented in exceptional cases [42-44]. The intensity blinking of the quantum dot can be visualized from the intensity trajectories that were recorded with a temporal resolution of $5 \mathrm{~ms}$ (Figure 1b). The breaks to true zero between trajectories are instrument-related, and indicate the change of the excitation wavelength, while the green line marks the background signal level without quantum dot emission, attributed to the emitter being trapped in a dark state.

Clearly, these drops to the background level are not related to narrow absorbance lines due to the band structure of the semiconductor quantum dots. In this case, drops in the recorded intensity would result in excitation wavelengths for which no emission can be recorded. The start and end of such a dark interval would then have to coincide with the start of a new excitation wavelength recording interval. We did not observe this behavior and the beginning and end of a dark period occurred stochastically.
The left panel of Figure $1 \mathrm{~b}$ depicts the intensity evolution for excitation from $522-524 \mathrm{~nm}$. For these wavelengths, the excitation spectrum showed strong intensity fluctuations. At $\lambda_{\text {ex }}=522 \mathrm{~nm}$, an intensity jump was observed immediately before the subsequent wavelength change. For $\lambda_{\mathrm{ex}}=523 \mathrm{~nm}$, the quantum dot was still in a non-emitting off-state, indicated by the signal intensity being at the background level. After $11.8 \mathrm{~s}$ it returned into a stable on-state after some initial short "bursts". Accordingly, transitions to short lasting off-states resulted in sudden dips in the excitation spectrum, and the intensity in the excitation spectrum did not drop to the background level, as the quantum dot was not dark during the whole integration interval.

The middle panel in Figure 1b depicts a decreased emission intensity that varied over time but did not drop to the background level. These variations in the observed emission intensity can be explained either as the result of fast blinking, below the time resolution of the experiment, or by transitions of the quantum dot to a dim (weakly-emitting) state [45]. As a result of this reduced emission we observed drops in the excitation spectrum over a number of wavelengths as can be seen around $550 \mathrm{~nm}$ in Figure 1a. Finally, we often found extended gaps in 
the excitation spectrum of single quantum dots as can be seen in the spectrum between $569 \mathrm{~nm}$ and $587 \mathrm{~nm}$ (Figure 1a). This gap in the excitation spectrum is attributed to a long lasting dark state followed by the return to an emitting state as can be seen in the right panel of Figure 1b. It is important to note that the observed drops in intensity do not correlate with changes in the excitation wavelength. Clearly, the observed drops and gaps in the spectrum would vanish if exclusively emitting states were sampled for each excitation wavelength. For detection intervals where a transition to or from a dark state occurred (e.g., Figure 1b, right panel) this correction can easily be performed by considering just the higher emission intensity level. Drops and gaps in the spectrum originating from dim states or very fast transitions below the sampling resolution, and from long lasting dark states longer than the integration time per excitation wavelength, could be avoided by repeated, possibly faster, scanning of the excitation wavelength, which requires further technical development for implementation in future studies.

We argue that the observed transitions between on- and offstates reflect the intrinsic emission characteristics inherent to individual quantum dots. In addition to the intensity blinking observed, we further confirmed that we were addressing a single emitter and thus single photons by analyzing the coincidence of detected photons in time using a Hanbury Brown and Twiss configuration [46]. The resulting photon-antibunching curves recorded in this manner exhibited a near zero correlation ( $\mathrm{g}^{2}=0.3$ ) for the detection of two photons at the same time, as shown for a typical example in Figure 1c. Photon-antibunching data give strong evidence for the observation of single emitters [47], since a single emitting system intrinsically cannot emit two photons at the same time. Generally, $\mathrm{g}^{2}$ values below 0.5 are accepted as a proof of single molecule observation [34].

The recorded spectra show varying degrees of blinking, ranging from spectra exhibiting almost no dips and gaps due to emission intermittencies (Figure 2a), to spectra where numerous transitions between emitting and dark states can be observed (Figure $2 \mathrm{~b}-\mathrm{d}$ ). The excitation spectrum shown in Figure 2a is very intense and shows only minor signs of blinking, and is based on the detection of $\sim 2 \cdot 10^{5}$ photons emitted from the sampled quantum dot. This number of detected photons is comparable to the average number of photons that can be detected from organic fluorophores $[48,49]$ and suggests that single emitter excitation spectroscopy could also be used for classes of emitters other than the very photostable quantum dots analyzed in this study.

The recorded data further enables the detailed analysis of the influence of the excitation wavelength on the blinking of single quantum dots. Numerous studies on single quantum dots have

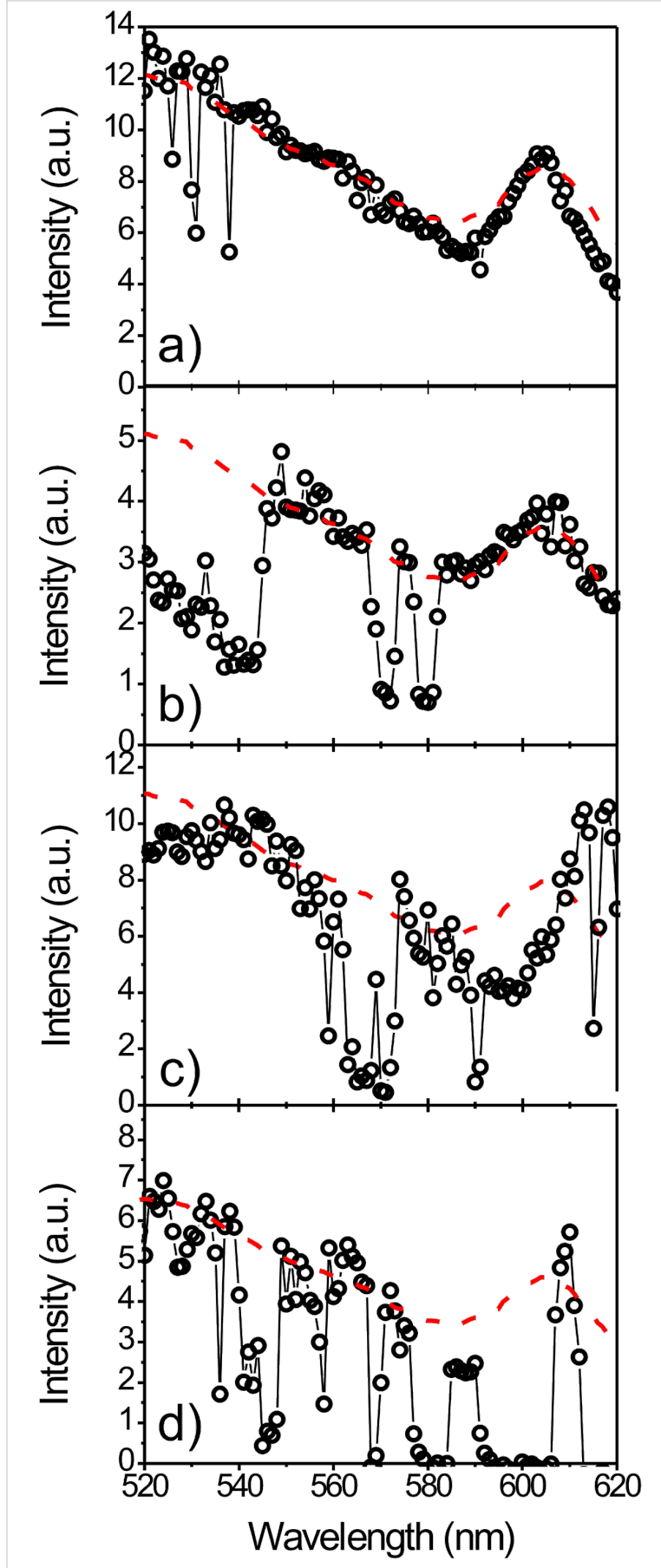

Figure 2: Single QD photoluminescence excitation spectra. For comparison, the ensemble excitation spectrum is shown as the red dashed line. The spectra show varying degrees of emission intermittencies visible as drops and gaps in the spectra. Especially in the wavelength region of the pronounced $1 \mathrm{~S}(\mathrm{e})-2 \mathrm{~S}_{3 / 2}(\mathrm{~h})$ transition the spectra show distinct differences between different quantum dots, reflecting the individual nature of each quantum dot.

shown complicated luminescence intermittency, or blinking, with power law statistics over many decades in time. In most of these studies a single excitation wavelength was used, and only recently has the first in-depth investigation of quantum dot 
blinking comparing a small number of different excitation wavelengths been published [32]. These studies were based on the statistical analysis of different quantum dots and an analysis of the emission of individual emitters using different excitation wavelengths has not yet been achieved. The approach presented here makes it possible to study the response of individual emitters to changes in excitation wavelength over a broad range. Hence, increased photoluminescence intermittency for certain excitation wavelengths will result in systematically reduced emission intensity for this wavelength in our study. We therefore calculated the sum of the photoluminescence excitation spectra from all single quantum dots analyzed in our study and compared it to the ensemble spectrum recorded with a calibrated ensemble spectrometer (Figure 3a). The summed photoluminescence excitation spectrum shows a number of interesting characteristics. Globally, blinking that was visible in the individual excitation spectra averages out, and the sum spectrum shows no explicit gaps where the intensity suddenly drops and then recurs. However, there are clear differences between the ensemble photoluminescence excitation spectrum and the summed excitation spectra from single quantum dots. On the short wavelength side, the sum spectrum declines much faster than the ensemble spectrum, while on the long wavelength side there is good agreement between the positions of the pronounced $1 \mathrm{~S}(\mathrm{e})-2 \mathrm{~S}_{3 / 2}(\mathrm{~h})$ transition. This discrepancy on the short wavelength side of the sum spectrum is not apparent in the individual spectra and can be understood from the details of how each single emitter excitation spectrum was recorded, that is, by considering that the excitation wavelength was always scanned from short to long wavelength. As the transition to dark states is driven by the excitation light, the probability to find a single quantum dot in a non-emitting state is minimal at the start of the experiment. Over time, which translates to longer excitation wavelengths in our experiment, the probability to find a quantum dot in a long-lived dark state increases. In the extreme case, a transition to a dark state occurs and photoluminescence is not regained before the end of the experiment (Figure 3a, inset). Thus, shorter excitation wavelengths are overweighted in the sum spectrum in the excitation scheme used, which was dictated by the monochromator used for these experiments (see Supporting Information File 1 for details) that only allows for scanning of the excitation from low to high wavelengths. One promising way to overcome this limitation in future experiments is to use an acousto-optical tunable filter (AOTF) for fast wavelength selection and bidirectional wavelength scanning.

However, not all discrepancies between the sum spectrum and the ensemble spectrum can be explained by the details of the excitation scheme used. If the probability for a transition to a long-lived dark state is independent of the excitation wave-

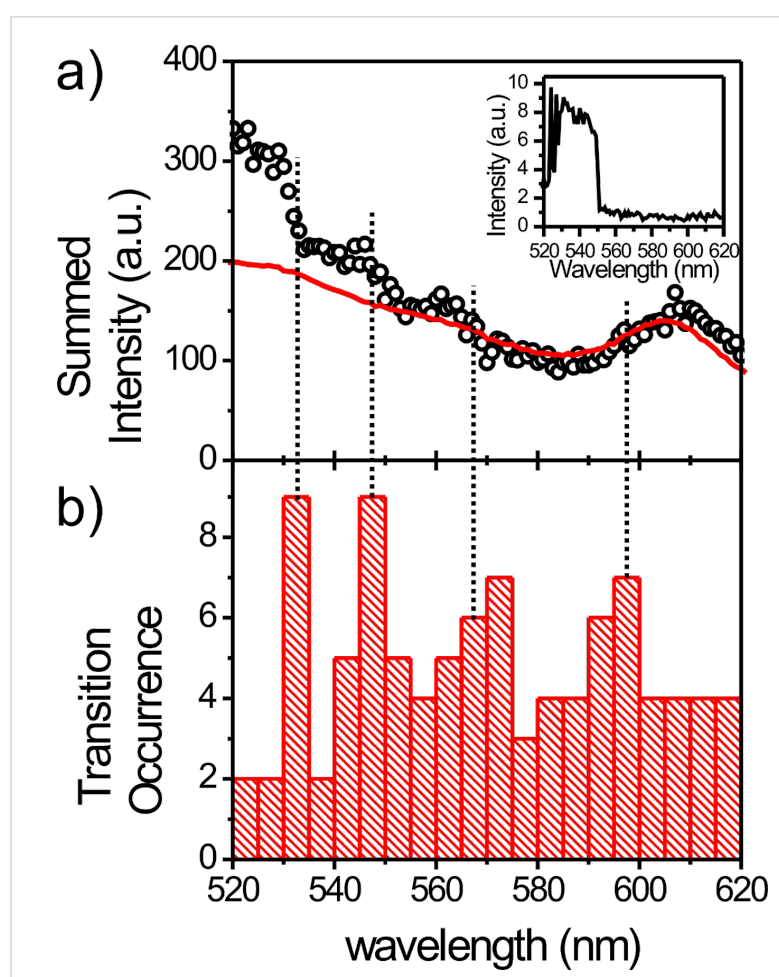

Figure 3: Single quantum dot excitation spectra reveal distinct excitation wavelengths with increased probability for a dark-state transition. a) sum spectrum of 48 excitation spectra of individual quantum dots (circles). Red: Ensemble excitation spectrum. Inset: Single quantum dot excitation spectra undergoing a transition to a dark state. b) Histogram of transitions to a long lived dark-state.

length, a steady decline of the summed single quantum dot excitation spectrum approaching the spectral shape of the ensemble spectrum is expected when both spectra are normalized to the long wavelength edges of the spectra. Indeed, we find good agreement between the sum and ensemble spectra for excitation wavelengths beyond $\sim 580 \mathrm{~nm}$, which suggests only a minor influence from transitions to dark states that do not recover during the entire data acquisition time in this wavelength range. On the other hand we find large deviations between the sum and ensemble spectra for excitation wavelengths below $\sim 580 \mathrm{~nm}$. This observation is consistent with reports that excitation in the band gap area results in little blinking compared to excitation above the band gap [32]. However, we do not see a smooth decline of the excitation sum spectrum, but observe what appear to be a number of steps $(\sim 530 \mathrm{~nm}, \sim 550 \mathrm{~nm}, 565 \mathrm{~nm}$, and $595 \mathrm{~nm}$ (less prominent); Figure 3a). These drops in the sum spectrum indicate that at the associated excitation wavelengths an increased probability of an intensity drop, that is, of a transition to a dark state, exists. The drops at $530 \mathrm{~nm}, 550 \mathrm{~nm}$ and $570 \mathrm{~nm}$ appear to be weakly reflected in the bulk spectrum. Since transitions to dark states are not sampled in the bulk spectrum due to the comparatively low excitation powers used to record bulk spectra, these similarities might point towards the 
molecular mechanism underlying the increased probability for a transition to a dark state. To verify that the observed steps in the sum spectrum indeed result from an increased number of single quantum dots changing to a dark state, we created a histogram of the wavelengths for which a transition to a dark state could be observed (Figure 3b). To estimate the statistical significance of the distribution obtained we determined the p-value assuming equal probability of a dark state transition for all power normalized excitation wavelengths. We obtain a p-value of 0.06 indicating a statistically significant result since there is only a low probability of $\sim 6 \%$ that the observed distribution originates from a random distribution of dark state transitions. The histogram shows significant peaks in the frequency of a dark state transition for the excitation wavelengths $\sim 532 \mathrm{~nm}$, $\sim 548 \mathrm{~nm}, \sim 570 \mathrm{~nm}$ and $\sim 595 \mathrm{~nm}$. All four peaks are correlated to a signature in the sum spectrum.

The data suggest that there is not only a large difference in the probability of a transition to a dark state for excitation in the band gap compared to excitation above the band gap, but that there are additionally certain excitation wavelengths which preferentially induce transitions to dark states. We exclude the idea of increased blinking rates for lower wavelengths due to increased absorbance and thereby a higher probability of an Auger assisted ionization, since the excitation powers used were smaller at lower wavelengths than at higher wavelengths. Additionally such a mechanism cannot explain the increased probability of a dark state transition for certain wavelengths only. Our data indicates that the formation of dark states shows a complex dependence on the excitation wavelengths used, suggesting that dark states can be reached via different pathways that can be accessed preferentially by using certain excitation wavelengths. Besides details on the wavelength dependent blinking of single emitters, our data also give access to the individual spectral properties of the quantum dots. In Figure 2 we show some typical examples of single quantum dot excitation spectra. As a guide to the eye and for comparison, the reference ensemble excitation spectrum is plotted as a dashed red line in each panel of Figure 2 .

Comparing the excitation spectra of different quantum dots we find both striking similarities and some clear differences between the spectra. The single quantum dot spectra are always enveloped by the ensemble spectrum below $\sim 580 \mathrm{~nm}$ (Figure 2, Figure 1a). Besides dips and gaps due to the blinking behavior on different timescales, we see no significant differences between the single quantum dots or distinct individual features in this part of the spectrum.

In general, observed wavelength dependent changes in photoluminescence can result from changes in either the absorbance or the photoluminescence quantum yield. Although excitation wavelength dependent changes in photoluminescence quantum efficiency have been discussed [50,51], Tonti et al. were able to show that there is no intrinsic deviation between the excitation and absorbance spectra of quantum dots once all corrections and sample handling are properly performed [52]. This result implies that the photoluminescence quantum yield in $\mathrm{CdSe}$ quantum dot ensembles is independent of the excitation wavelength, and that analyzing the excitation spectra also allows one to draw conclusions about the absorbance spectra of single quantum dots. Following from this, the observed behavior directly results from the differences in the absorbance at different wavelengths of the quantum dots. The spectral region below $580 \mathrm{~nm}$, where we find no clear signs of individual spectral behavior from individual quantum dots, is exactly that region where a quasicontinuum of optical transitions was observed at cryogenic temperatures, corresponding to low lying energy barriers between distinct states predicted by the theory. It is therefore not surprising that, except for blinking events, no individual characteristics of the observed quantum dots can be identified at room temperature in this wavelength region.

The picture changes significantly when looking at the pronounced transition at $\sim 605 \mathrm{~nm}$, closer to the band edge, where we find clear differences in the shape, height and spectral position of this peak in the individual excitation spectra. Variations in the spectral position of this peak from individual quantum dots are attributed to differences in the size of the individual quantum dots. As expected for a single molecule study, we also find that the width of the transition is generally smaller than the width of the ensemble transition. This broadening in the ensemble or summed single molecule spectra results from the superposition of a large number of excitation spectra of single quantum dots of varying peak wavelength. Interestingly, we find some excitation spectra that do not show narrowed spectral features, e.g., Figure 2b, pointing towards the existence of a phenomenon equivalent to spectral emission diffusion $[53,54]$ in the excitation spectrum and, by inference, in the absorbance. Especially since we only find minor variations in the excitation spectra at the short wavelength side below $580 \mathrm{~nm}$ and do not observe individual fingerprints in this area, the observation of not only the spectral position but also the different relative height and shape for the $1 \mathrm{~S}(\mathrm{e})-2 \mathrm{~S}_{3 / 2}(\mathrm{~h})$ transition reflects the individual characteristics of each single quantum dot. Moreover, our spectra strikingly show that the different heights and shapes of the transitions are not correlated with their spectral position and hence with the particle size, suggesting different molecular origins. The shape and height of the peak from the $1 \mathrm{~S}(\mathrm{e})-2 \mathrm{~S}_{3 / 2}(\mathrm{~h})$ transition is determined by the coupling between these states. It has been shown that transitions close to the band edge show a fine structure splitting into 
sublevels due to the quantum dot crystal field, shape anisotropy, and confinement enhanced electron-hole exchange interactions [55]. Although considerably broadened at room temperature and hence invisible in the ensemble, the variation of the height and shape of the peak around $605 \mathrm{~nm}$, which we have observed for individual quantum dots, reflects details of these transitions. Since the differences in relative height of spectral features in the photoluminescence excitation spectrum represent the strength of the coupling between the ground and excited state of the individual quantum dots, it is likely that the observed differences in the individual excitation spectra reflect the differences in the photoluminescence quantum efficiency of individual quantum dots, as previously reported [56,57].

\section{Conclusion}

We have recorded for the first time single molecule excitation spectra at room temperature. The required spectrally narrow excitation over a wide spectral range of $100 \mathrm{~nm}$ was realized by using a monochromator to select the excitation wavelength from a supercontinuum white light source. The suitability of our approach and its potential were demonstrated by studying single quantum dots. The single emitter nature of our quantum dot samples was confirmed by photon antibunching experiments. Analysis of the single quantum dot excitation spectra gave access to hitherto unexplored details of this class of emitters. For the CdSe nanocrystals investigated, we found strong indications for an increased probability for a transition to long-lived dark states at specific excitation wavelengths, suggesting that these wavelengths are unsuitable for single photon applications. The excitation spectra showed no clear individual features for excitation wavelengths well above the band gap, but exhibited large differences for transitions close to the excitonic peak, representing the fine structure splitting into energetic sublevels of the individual quantum dots. Further, we found variations in the width and the spectral position of transitions for individual quantum dots.

On the basis of these investigations of quantum dots, we expect single molecule excitation spectroscopy to become a valuable addition to the established single molecule spectroscopy methods. The approach will not only aid in the analysis of isolated dyes or nanoparticles but also prove valuable in analyzing complex emitting systems such as Förster resonance energy transfer (FRET) pairs, fluorescent proteins and upconversion particles.

\section{Experimental}

We realized an instrument capable of single molecule excitation spectroscopy by combining a high power supercontinuum white light source (Fianium SC-400pp) with a grating spectrometer (Spectra Pro 300i, Acton Research) for excitation wave- length selection and a custom built confocal scanning stage single molecule detection microscope (for details see Supporting Information File 1). Experiments were carried out in two steps. First, the quantum dots immobilized in a thin polymer layer at low concentration $\left(c_{\mathrm{QD}}=5 \times 10^{-10} \mathrm{M}\right)$ were visualized by creating a raster scanned emission intensity image of an $20 \times 20 \mu \mathrm{m}^{2}$ area of the sample using a fixed excitation wavelength $\left(\lambda_{\mathrm{ex}}=600 \mathrm{~nm}\right)$. After localization, the single quantum dots were positioned in the laser focus and the excitation wavelength was swept from $520 \mathrm{~nm}$ to $620 \mathrm{~nm}$ in increments of $1 \mathrm{~nm}$. For each excitation wavelength the emitted fluorescence intensity was recorded for $400 \mathrm{~ms}$, followed by an increment in the excitation wavelength during which data acquisition was disabled ( $\sim 70 \mathrm{~ms}$ duration). In this way we obtained $400 \mathrm{~ms}$ fluorescence intensity trajectories depicting the evolution of the emission intensity for each excitation wavelength, interspersed with $\sim 70 \mathrm{~ms}$ zero-intensity periods representing the excitation wavelength increment (Figure 1). To obtain the fluorescence excitation spectrum, the total emission intensity per excitation wavelength was integrated and calibrated using a reference spectrum recorded for a film of emitters (i.e., an ensemble of emitters) to compensate for wavelength dependent excitation power and detection efficiency (for details see Supporting Information File 1).

\section{Supporting Information}

Supporting Information features detailed description of instrumentation, and data acquisition and correction procedures.

\section{Supporting Information File 1}

Experimental details.

[http://www.beilstein-journals.org/bjnano/content/

supplementary/2190-4286-2-56-S1.pdf]

\section{Acknowledgements}

This work was financed by a strategic research orientation grant from the MESA+ Institute for Nanotechnology to CB, MS, and VS and an MWK grant of the Land Baden-Württemberg to FS, SP, MS and AJM.

\section{References}

1. Moerner, W. J. Phys. Chem. B 2002, 106, 910-927. doi:10.1021/jp012992g

2. Schleifenbaum, F.; Blum, C.; Subramaniam, V.; Meixner, A. J. Mol. Phys. 2009, 107, 1923-1942. doi:10.1080/00268970802635004

3. Tinnefeld, P.; Sauer, M. Angew. Chem., Int. Ed. 2005, 44, 2642-2671. doi:10.1002/anie.200300647

4. Lupton, J. M. Adv. Mater. 2010, 22, 1689-1721. doi:10.1002/adma.200902306 
5. Woell, D.; Uji-I, H.; Schnitzler, T.; Hotta, J. I.; Dedecker, P.; Herrmann, A.; De Schryver, F. C.; Muellen, K.; Hofkens, J. Angew. Chem., Int. Ed. 2008, 47, 783-787. doi:10.1002/anie.200704196

6. Roeffaers, M.; Sels, B.; Uji-i, H.; De Schryver, F.; Jacobs, P.; De Vos, D.; Hofkens, J. Nature 2006, 439, 572-575. doi:10.1038/nature04502

7. Schuler, B. ChemPhysChem 2005, 6, 1206-1220. doi:10.1002/cphc.200400609

8. Blum, C.; Subramaniam, V. Anal. Bioanal. Chem. 2009, 393, 527-541. doi:10.1007/s00216-008-2425-x

9. Dertinger, T.; Pacheco, V.; von der Hocht, I.; Hartmann, R.; Gregor, I.; Enderlein, J. ChemPhysChem 2007, 8, 433-443. doi:10.1002/cphc.200600638

10. Michalet, X.; Pinaud, F.; Bentolila, L.; Tsay, J.; Doose, S.; Li, J.; Sundaresan, G.; Wu, A.; Gambhir, S.; Weiss, S. Science 2005, 307, 538. doi:10.1126/science.1104274

11. Moerner, W. E. Proc. Natl. Acad. Sci. U. S. A. 2007, 104, 12596-12602. doi:10.1073/pnas.0610081104

12. Forkey, J. N.; Quinlan, M. E.; Goldman, Y. E. Prog. Biophys. Mol. Biol. 2000, 74, 1-35. doi:10.1016/S0079-6107(00)00015-8

13. Peterman, E.; Brasselet, S.; Moerner, W. J. Phys. Chem. A 1999, 103 , 10553-10560. doi:10.1021/jp991968o

14. Schleifenbaum, F.; Blum, C.; Elgass, K.; Subramaniam, V.; Meixner, A. J. J. Phys. Chem. B 2008, 112, 7669-7674. doi:10.1021/jp7114753

15. Kapusta, P.; Wahl, M.; Benda, A.; Hof, M.; Enderlein, J. J. Fluoresc. 2007, 17, 43-48. doi:10.1007/s10895-006-0145-1

16. Tinnefeld, P.; Buschmann, V.; Herten, D.; Han, K.; Sauer, M. Single Mol. 2000, 1, 215-223. doi:10.1002/1438-5171(200009)1:3<215::AID-SIMO215>3.0.CO;2-S

17. Blum, C.; Meixner, A. J.; Subramaniam, V. ChemPhysChem 2008, 9 , 310-315. doi:10.1002/cphc.200700784

18. Cotlet, M.; Hofkens, J.; Habuchi, S.; Dirix, G.; van Guyse, M.; Michiels, J.; Vanderleyden, J.; de Schryver, F. C. Proc. Natl. Acad. Sci. U. S. A. 2001, 98, 14398-14403. doi:10.1073/pnas.251532698

19. Lacoste, T. D.; Michalet, X.; Pinaud, F.; Chemla, D. S.; Alivisatos, A. P.; Weiss, S. Proc. Natl. Acad. Sci. U. S. A. 2000, 97, 9461-9466. doi:10.1073/pnas. 170286097

20. Blum, C.; Meixner, A. J.; Subramaniam, V. Biophys. J. 2004, 87, 4172-4179. doi:10.1529/biophysj.104.049452

21. Widengren, J.; Kudryavtsev, V.; Antonik, M.; Berger, S.; Gerken, M.; Seidel, C. A. M. Anal. Chem. 2006, 78, 2039-2050. doi:10.1021/ac0522759

22. Eckel, R.; Walhorn, V.; Pelargus, C.; Martini, J.; Enderlein, J.; Nann, T.; Anselmetti, D.; Ros, R. Small 2007, 3, 44-49. doi:10.1002/smll.200600130

23. Taminiau, T.; Stefani, F.; Segerink, F.; Van Hulst, N. Nat. Photonics 2008, 2, 234-237. doi:10.1038/nphoton.2008.32

24. Chizhik, A.; Schleifenbaum, F.; Gutbrod, R.; Chizhik, A.; Khoptyar, D.; Meixner, A. J.; Enderlein, J. Phys. Rev. Lett. 2009, 102, 073002. doi:10.1103/PhysRevLett.102.073002

25. Gaiduk, A.; Yorulmaz, M.; Ruijgrok, P. V.; Orrit, M. Science 2010, 330, 353-356. doi:10.1126/science.1195475

26. Chong, S.; Min, W.; Xie, X. S. J. Phys. Chem. Lett. 2010, 1 , 3316-3322. doi:10.1021/jz1014289

27. Kukura, P.; Celebrano, M.; Renn, A.; Sandoghdar, V. J. Phys. Chem. Lett. 2010, 1, 3323-3327. doi:10.1021/jz101426x
28. Orrit, M.; Bernard, J. Phys. Rev. Lett. 1990, 65, 2716-2719. doi:10.1103/PhysRevLett.65.2716

29. Ambrose, W.; Moerner, W. Nature 1991, 349, 225-227. doi:10.1038/349225a0

30. Kroutvar, M.; Ducommun, Y.; Heiss, D.; Bichler, M.; Schuh, D.; Abstreiter, G.; Finley, J. Nature 2004, 432, 81-84. doi:10.1038/nature03008

31. Neuhauser, R.; Shimizu, K.; Woo, W.; Empedocles, S.; Bawendi, M. Phys. Rev. Lett. 2000, 85, 3301-3304. doi:10.1103/PhysRevLett.85.3301

32. Knappenberger, K., Jr.; Wong, D.; Romanyuk, Y.; Leone, S. Nano Lett. 2007, 7, 3869-3874. doi:10.1021/nl0714740

33. Norris, D. J.; Bawendi, M. G. Phys. Rev. B 1996, 53, 16338-16346. doi:10.1103/PhysRevB.53.16338

34. Zwiller, V.; Blom, H.; Jonsson, P.; Panev, N.; Jeppesen, S.; Tsegaye, T.; Goobar, E.; Pistol, M.; Samuelson, L.; Björk, G. Appl. Phys. Lett. 2001, 78, 2476. doi:10.1063/1.1366367

35. Loss, D.; DiVincenzo, D. Phys. Rev. A 1998, 57, 120-126. doi:10.1103/PhysRevA.57.120

36. Medintz, I. L.; Uyeda, H. T.; Goldman, E. R.; Mattoussi, H. Nat. Mater. 2005, 4, 435-446. doi:10.1038/nmat1390

37. Alivisatos, A. P.; Gu, W. W.; Larabell, C. Annu. Rev. Biomed. Eng 2005, 7, 55-76. doi:10.1146/annurev.bioeng.7.060804.100432

38. Ward, M.; Farrow, T.; See, P.; Yuan, Z.; Karimov, O.; Bennett, A.; Shields, A.; Atkinson, P.; Cooper, K.; Ritchie, D. Appl. Phys. Lett. 2007, 90, 063512. doi:10.1063/1.2472172

39. Klimov, V. I. Annu. Rev. Phys. Chem. 2007, 58, 635-673. doi:10.1146/annurev.physchem.58.032806.104537

40. Empedocles, S.; Norris, D.; Bawendi, M. Phys. Rev. Lett. 1996, 77, 3873-3876. doi:10.1103/PhysRevLett.77.3873

41. Htoon, H.; Cox, P. J.; Klimov, V. I. Phys. Rev. Lett. 2004, 93, 187402. doi:10.1103/PhysRevLett.93.187402

42. Wang, X.; Ren, X.; Kahen, K.; Hahn, M.; Rajeswaran, M.; Maccagnano-Zacher, S.; Silcox, J.; Cragg, G.; Efros, A.; Krauss, T. Nature 2009, 459, 686-689. doi:10.1038/nature08072

43. Efros, A. Nat. Mater. 2008, 7, 612-613. doi:10.1038/nmat2239

44. Hohng, S.; Ha, T. J. Am. Chem. Soc. 2004, 126, 1324-1325. doi:10.1021/ja039686w

45. Fisher, B. R.; Eisler, H.-J.; Stott, N. E.; Bawendi, M. G. J. Phys. Chem. B 2004, 108, 143-148. doi:10.1021/jp035756+

46. Basche, T.; Moerner, W. E.; Orrit, M.; Talon, H. Phys. Rev. Lett. 1992, 69, 1516-1519. doi:10.1103/PhysRevLett.69.1516

47. Lounis, B.; Bechtel, H.; Gerion, D.; Alivisatos, P.; Moerner, W. Chem. Phys. Lett. 2000, 329, 399-404. doi:10.1016/S0009-2614(00)01042-3

48. Lord, S. J.; Lee, H.-I. D.; Samuel, R.; Weber, R.; Liu, N.; Conley, N. R.; Thompson, M. A.; Twieg, R. J.; Moerner, W. E. J. Phys. Chem. B 2010, 114, 14157-14167. doi:10.1021/jp907080r

49. Deschenes, L. A.; Vanden Bout, D. A. Chem. Phys. Lett. 2002, 365, 387-395. doi:10.1016/S0009-2614(02)01490-2

50. Hoheisel, W. J. Chem. Phys. 1994, 101, 845.

51. Rumbles, G.; Selmarten, D.; Ellingson, R.; Blackburn, J.; Yu, P.; Smith, B.; Mićić, O.; Nozik, A. J. Photochem. Photobiol., A 2001, 142, 187-195. doi:10.1016/S1010-6030(01)00513-5

52. Tonti, D.; Van Mourik, F.; Chergui, M. Nano Lett. 2004, 4, 2483-2487. doi:10.1021/nl0486057

53. Stracke, F.; Blum, C.; Becker, S.; Müllen, K.; Meixner, A. J. ChemPhysChem 2005, 6, 1242-1246. doi:10.1002/cphc.200400436 
54. Stracke, F.; Blum, C.; Becker, S.; Müllen, K.; Meixner, A. J. Chem. Phys. Lett. 2000, 325, 196-202.

doi:10.1016/S0009-2614(00)00633-3

55. Htoon, H.; Takagahara, T.; Kulik, D.; Baklenov, O.; Holmes, A., Jr.; Shih, C. Phys. Rev. Lett. 2002, 88, 087401.

doi:10.1103/PhysRevLett.88.087401

56. Biebricher, A.; Sauer, M.; Tinnefeld, P. J. Phys. Chem. B 2006, 110, 5174-5178. doi:10.1021/jp060660b

57. Brokmann, X.; Coolen, L.; Dahan, M.; Hermier, J. P. Phys. Rev. Lett 2004, 93, 107403. doi:10.1103/PhysRevLett.93.107403

\section{License and Terms}

This is an Open Access article under the terms of the Creative Commons Attribution License

(http://creativecommons.org/licenses/by/2.0), which permits unrestricted use, distribution, and reproduction in any medium, provided the original work is properly cited.

The license is subject to the Beilstein Journal of

Nanotechnology terms and conditions:

(http://www.beilstein-journals.org/bjnano)

The definitive version of this article is the electronic one which can be found at: doi:10.3762/bjnano. 2.56 